\title{
A GENERALIZED LAX-MILGRAM THEOREM
}

\author{
EDWARD M. LANDESMAN
}

1. Introduction. In [2], the author introduced the Hilbert spaces $H_{A}$ and $H_{A^{\prime}}$, which were associated with an operator $A$ and its *-reciprocal $A^{\prime} \equiv A^{*-1}$. Some general theorems relating these spaces to each other were formulated and proven.

These Hilbert spaces proved to be those which are of prime importance when studying partial differential equations, in particular linear elliptic partial differential equations. The author has shown in [2] that when $A$ is the operator $G_{k}$, that is, the operator which maps a function into itself and its first $k$-derivatives, then $H_{G_{k}}$ and $H_{G_{k^{\prime}}}$ are the spaces which form the "natural" setting for the study of existence and regularity theory.

In this paper the author generalizes in a natural way the LaxMilgram Theorem [3] by proving the theorem for the more generalized case when $H_{A}$ and $H_{A}$, are the underlying spaces. The method of proof follows that employed in [3].

2. Preliminaries. Let $H$ and $H^{1}$ be any two real Hilbert spaces. Suppose $A$ is an operator (closed and dense linear transformation) from $H$ into $H^{1}$. For simplicity, we take the null space $N_{A}$ of the operator $A$ to be zero.

Suppose that the domain $D_{A}$ of $A$ is completed with respect to the range norm $\|A x\|$. Designate this by

$$
\|x\|_{A}=\|A x\|
$$

for all $x$ in the domain $D_{A}$ of $A$. We call the Hilbert space so obtained $H_{A}$.

Consider now the closure of the range of $A, \bar{R}_{A}$. This is a Hilbert space with norm being the natural norm in $H^{1}$.

Clearly, the operator $A$ is a norm preserving map of $D_{A}$ onto $R_{A}$ where (2.1) is taken as the norm for $D_{A}$.

We extend $A$ linearly to a unitary operator on $H_{A}$ onto $\bar{R}_{A}$. We call this extension $\tilde{A}$.

Similarly, we complete the domain $D_{A^{\prime}}$ of $A^{\prime} \equiv A^{*-1}$, with respect to the norm

$$
\|x\|_{A^{\prime}}=\left\|A^{\prime} x\right\|
$$

for all $x$ in the domain $D_{A^{\prime}}$ of $A^{\prime}$. We call this Hilbert space $H_{A^{\prime}}$.

Received by the editors January 2, 1967. 
As was shown in [1], the completion of the domain $D_{A^{\prime}}$ with respect to the norm (2.2) is equivalent to completing $D_{A^{\prime}}$ with respect to the norm

$$
\|x\|_{A^{\prime}}=\operatorname{Sup}_{\boldsymbol{\nu} \in D_{\mathbf{A}}} \frac{(x, y)}{\|A y\|}
$$

where $(x, y)$ denotes the ordinary inner product in $H$.

We call $\tilde{A}^{\prime}$ the unitary operator which maps $H_{A^{\prime}}$ onto $\bar{R}_{A^{\prime}}$. As an immediate result, we have:

Lemma 2.1. The closure of the range of $A$ is equal to the closure of the range of $A^{\prime}$, i.e., $\bar{R}_{A}=\bar{R}_{A^{\prime}}$.

The result follows from the following equalities

$$
\begin{aligned}
\left(\bar{R}_{\boldsymbol{A}}\right)^{\perp} & =R_{\boldsymbol{A}}^{\perp}=N_{A^{-1}}=N_{\boldsymbol{A}^{*}} \\
& =R_{\mathbf{A}^{\prime}}^{\perp}=\left(\bar{R}_{A^{\prime}}\right)^{\perp} .
\end{aligned}
$$

Since $\bar{R}_{A}$ and $\bar{R}_{A^{\prime}}$ are closed, we have

$$
\bar{R}_{A}=\left[\left(\bar{R}_{\boldsymbol{A}}\right)^{\perp}\right]^{\perp}=\left[\left(\bar{R}_{\boldsymbol{A}^{\prime}}\right)^{\perp}\right]^{\perp}=\bar{R}_{\boldsymbol{A}^{\prime}}
$$

As a simple consequence of Lemma 2.1 , we see that the operator $\tilde{A}^{\prime}$ maps $H_{A^{\prime}}$ on to $\bar{R}_{A}$.

We remark that by the way we have constructed $H_{A}$ and $H_{A^{\prime}}$, we may always choose an $x$ in $H_{A}$ such that:

$$
\tilde{A} x=\tilde{A}^{\prime} z \text { for a given } z \text { in } H_{A^{\prime}} .
$$

This enables us to define an "inner product" between the elements of $H_{A}$ and the elements of $H_{A^{\prime}}$. We do so as follows:

If $x$ is in $H_{A}$ and $y$ is in $H_{A^{\prime}}$, define their inner product as

$$
\langle x, y\rangle=\left(\tilde{A} x, \tilde{A}^{\prime} y\right) \text {. }
$$

We note that if $x$ is in $D_{A}$ and $y$ is in $D_{A^{\prime}}$, then $\langle x, y\rangle=(x, y)$, the ordinary inner product in $H$. Henceforth, let \|\|$_{H_{\boldsymbol{A}}}$ and \|\|$_{H_{\boldsymbol{A}^{\prime}}}$ denote the norms in $H_{A}$ and $H_{A^{\prime}}$ respectively. We immediately obtain the following theorems which have been proven in [2].

Theorem 2.1 (Generalized Schwarz Inequality). If $x$ is in $H_{A}$, $y$ is in $H_{A^{\prime}}$, then

$$
|\langle x, y\rangle| \leqq\|x\|_{H_{A}}\|y\|_{H_{A^{\prime}}} .
$$

Theorem 2.2 (Representation Theorem). Every bounded linear functional $L(x)$ over $H_{A}$ can be represented as 


$$
L(x)=\langle x, y\rangle
$$

where $y$ is in $H_{A^{\prime}}$.

3. Main theorem. Let $H$ and $H^{1}$ be real Hilbert spaces. Let $A$ be an operator from $H$ into $H^{1}$ and define the Hilbert spaces $H_{\boldsymbol{A}}$ and $H_{\boldsymbol{A}^{\prime}}$ as in $\S 2$. Suppose $B(x, y)$ is a bilinear functional on $H_{A} \times H_{A^{\prime}}$, i.e., $x$ is in $H_{A}$ and $y$ is in $H_{A^{\prime}}$.

Theorem 3.1 (Generalized Lax-Milgram Theorem). Suppose that $B(x, y)$ is bounded in the following sense

$$
|B(x, y)| \leqq K\|x\|_{H_{\mathbf{A}}}\|y\|_{H_{\mathbf{A}^{\prime}}}
$$

for some positive constant $K$, and for all $x$ in $H_{A}$ and for all $y$ in $H_{A^{\prime}}$.

Furthermore, suppose that there is an $M>0$ such that

$$
B(x, y) \geqq M\|y\|_{H_{\mathbf{A}^{\prime}}}^{2}
$$

for all $x$ and $y$ such that $\tilde{A} x=\tilde{A}^{\prime} y$.

Then given any bounded linear functional $L(x)$ over $H_{A}$, there exists a unique $y$ in $H_{A^{\prime}}$ such that

$$
L(x)=B(x, y) \text { for all } x \text { in } H_{A} .
$$

Proof. Assuming the existence of $y$, we first prove that $y$ is unique.

If $y$ is not unique, then there is a $y_{1}$ in $H_{A^{\prime}}$ such that:

$$
B(x, y)=B\left(x, y_{1}\right) \text {, i.e., } \quad B\left(x, y-y_{1}\right)=0 \text { for all } x \text { in } H_{\Delta} .
$$

Since the above is true for all $x$ in $H_{A}$, by the way we have constructed $H_{A}$ and $H_{A^{\prime}}$, we may choose an $x$ in $H_{A}$, say $x_{0}$, such that

$$
\tilde{A} x_{0}=\tilde{A}^{\prime}\left(y-y_{1}\right) \text {. }
$$

From condition (3.2), and (3.4), we get

$$
M\left\|y-y_{1}\right\|_{H_{A^{\prime}}}^{2} \leqq B\left(x_{0}, y-y_{1}\right)=0 \text { i.e., } \quad y=y_{1} .
$$

In order to prove the existence portion of the theorem, we employ a sequence of lemmas similar to what Lax and Milgram do in [3].

Define a subset $S$ of $H_{A}$, as follows:

$S$ is the set of all $y$ in $H_{A^{\prime}}$ for which there is a $z$ in $H_{A^{\prime}}$ such that:

$$
\langle x, y\rangle=B(x, z) \text { for all } x \text { in } H_{A} \text {. }
$$

Lemma 3.1. The set $S$ is a subspace of $H_{A^{\prime}}$.

Let $y_{1}$ and $y_{2}$ be in $H_{A^{\prime}}$. Then $\left\langle x, y_{1}\right\rangle=B\left(x, z_{1}\right)$ for some $z_{1}$ in $H_{A^{\prime}}$ and $\left\langle x, y_{2}\right\rangle=B\left(x, z_{2}\right)$ for some $z_{2}$ in $H_{A^{\prime}}$. 
These two equations yield $\left\langle x, y_{1}-y_{2}\right\rangle=B\left(x, z_{1}-z_{2}\right)$. This shows that $y_{1}-y_{2}$ is in $H_{A^{\prime}}$.

If $y$ is in $H_{A^{\prime}}$ and $r$ is any real number, then from

$$
\langle x, y\rangle=B(x, z) \text { for some } z \text { in } H_{A^{\prime}}
$$

it follows that $\langle x, r y\rangle=B(x, r z)$ i.e., $r y$ is in $H_{A^{\prime}}$.

Lemma 3.2. The element $z$ is bounded with respect to the element $y$.

By the way $S$ is defined, if $y$ is in $S$, there is a $z$ in $H_{A^{\prime}}$ such that:

$$
\langle x, y\rangle=B(x, z) \text { for all } x \text { in } H_{\boldsymbol{A}} .
$$

Choose $x$ such that:

$$
\tilde{A} x=\tilde{A}^{\prime} z .
$$

Then by (3.2),

$$
\langle x, y\rangle=B(x, z) \geqq M\|z\|_{H_{\mathbf{A}^{\prime}}}^{2} .
$$

Applying the Generalized Schwarz inequality (Theorem 2.1) to the left side of (3.8), we obtain:

$$
\|x\|_{H_{\Delta}}\|y\|_{H_{\Delta^{\prime}}} \geqq B(x, z) \geqq M\|z\|_{H_{\mathbf{A}^{\prime}}}^{2} .
$$

But by (3.7), $\|x\|_{H_{A}} \equiv\|\tilde{A} x\|=\left\|\tilde{A}^{\prime} z\right\| \equiv\|z\|_{H_{A^{\prime}}}$, therefore (3.9) becomes

$$
\|z\|_{H_{\mathbf{A}^{\prime}}}\|y\|_{H_{A^{\prime}}} \geqq B(x, z) \geqq M\|z\|_{H_{\mathbf{A}^{\prime}}}^{2} .
$$

Dividing the left and right side of (3.10) by $M\|z\|_{H_{A^{\prime}}}$, we obtain the result:

$$
\|z\|_{H_{\mathbf{A}^{\prime}}} \leqq(1 / M)\|y\|_{H_{\mathbf{A}^{\prime}}}
$$

Lemma 3.3. The set $S$ is closed.

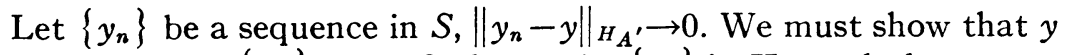
is in $S$. Since the $\left\{y_{n}\right\}$ are in $S$, there exist $\left\{z_{n}\right\}$ in $H_{A^{\prime}}$ such that:

$$
\left\langle x, y_{n}\right\rangle=B\left(x, z_{n}\right) \text { for all } x \text { in } H_{A} \text {. }
$$

By Lemma 3.2,

$$
\left\|z_{n}-z_{m}\right\|_{H_{A^{\prime}}} \leqq(1 / M)\left\|y_{n}-y_{m}\right\|_{H_{A^{\prime}}} .
$$

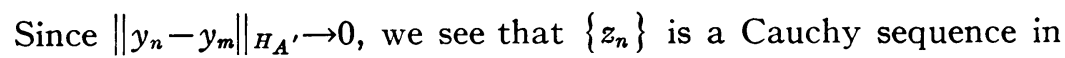
$H_{A^{\prime}}$, and hence converges to some $z$ in $H_{A^{\prime}}$.

Since by (3.1), $B(x, y)$ is continuous in both arguments, we have: $\langle x, y\rangle=\left\langle x, \lim _{n} y_{n}\right\rangle=B\left(x, \lim _{n} z_{n}\right)=B(x, z)$ for all $x$ in $H_{A}$, i.e., $y$ is in $S$ and $S$ is closed. 
We are now in a position to prove our theorem. It remains to show that $S$ is all of $H_{A^{\prime}}$. This will show that all "inner products" of $H_{A} \times H_{A^{\prime}}$ can be represented as an inner product with respect to $B$. By the Representation Theorem (Theorem 2.2), since every bounded linear functional $L(x)$ over $H_{A}$ can be represented as an "inner product" over $H_{A} \times H_{A^{\prime}}$, we will be through.

Suppose that $S$ is not all of $H_{A^{\prime}}$. Since $S$ is a closed subspace of $H_{A^{\prime}}$, write

$$
H_{A^{\prime}}=S \oplus S^{\perp}
$$

where $S^{\perp}$ is nonempty and orthogonal to all of $S$. Let $y_{0} \neq 0$ be in $S^{\perp}$.

Consider $B\left(x, y_{0}\right)$ which is a bounded linear functional of $x$ since $y_{0}$ is fixed. By the Representation Theorem (Theorem 2.2), there exists a $y$ in $H_{A^{\prime}}$ such that:

$$
B\left(x, y_{0}\right)=\langle x, y\rangle
$$

for all $x$ in $H_{A}$, i.e., $y$ is in $S$.

Choose $x$ such that

$$
\tilde{A} x=\tilde{A}^{\prime} y_{0} .
$$

Then by (3.2), and (3.11), we have

$$
M\left\|y_{0}\right\|_{H_{A^{\prime}}}^{2} \leqq B\left(x, y_{0}\right)=\langle x, y\rangle .
$$

By our choice of $x$, the last term of the above inequality becomes:

$$
\langle x, y\rangle=\left(\tilde{A} x, \tilde{A}^{\prime} y\right)=\left(\tilde{A}^{\prime} y_{0}, \tilde{A}^{\prime} y\right)=\left(y_{0}, y\right)_{H_{\mathbf{A}^{\prime}}}=0,
$$

since $y$ is in $S$ and $y_{0}$ is in $S^{\perp}$.

We have therefore from (3.12) that

$$
M\left\|y_{0}\right\|_{H_{A^{\prime}}}^{2} \leqq 0
$$

i.e., $y_{0}=0$, and the proof is complete.

Corollary (Lax-Milgram Theorem). Let $H$ be a real Hilbert space and $B(x, y)$ a bilinear functional which is bounded, i.e., $|B(x, y)|$ $\leqq K\|x\|\|y\|$ and positive definite, i.e., there is a constant $h>0$ such that $B(x, x) \geqq h\|x\|^{2}$ for all $x$ in $H$. Then given any bounded linear functional $L(x)$ on $H$, there is a unique $y$ in $H$ such that $L(x)=B(x, y)$.

In Theorem 3.1, choose $H=H^{1}$. Take $A=A^{\prime}=I$, the identity operator. Then $H_{A}=H_{A^{\prime}}=H$, and the corollary is an immediate special case of Theorem 3.1.

REMARK. If $A$ is the operator $G_{k}$, which maps a function into itself and its first $k$ derivatives, see [2], then $A^{\prime}=G_{k^{\prime}}$ and the spaces $H_{G_{k}}$ 
and $H_{G_{k^{\prime}}}$ are the spaces which are of ten used in elliptic partial differential equations. In fact $H_{G_{k}}$ is the space $H_{k}$, which is the completion of the $C^{\infty}$ functions under the $k$-Dirichlet norm, while $H_{G_{k^{\prime}}}$ is the so called $H_{-k}$ space which comes into play when using the negativenorms defined by P. Lax, see [4]. The author relates these concepts in $[2]$.

With the above choice of $A=G_{k}$, our theorem says that any bounded linear functional over $H_{k}$ is representable as a bilinear functional over $H_{k} \times H_{-k}$.

\section{BiBLIOGRAPHY}

1. M. R. Hestenes, Applications of the theory of quadratic forms in Hilbert space to the calculus of variations, Pacific J. Math. 1 (1951), 525-581.

2. E. M. Landesman, Hilbert-space methods in elliptic partial differential equations, Pacific J. Math. 21 (1967), 113-131.

3. P. D. Lax and A. N. Milgram, Parabolic equations, Annals of Mathematics Studies No. 33, Princeton Univ. Press, Princeton, N. J., 1954.

4. P. D. Lax, On Cauchy's problem for hyperbolic equations and the differentiability of solutions of elliptic equations, Comm. Pure Appl. Math. 8 (1955), 615-633.

University of California, Santa Cruz 